hospitals with heliport outside the hospital $(5 \pm 2.18$ and 6 \pm 2.94 vs. $9 \pm 3.94$ and $12 \pm 3.49, \mathrm{p}<0.001$ ).

Conclusion Hospital based heliports ensure shorter time delay from landing to pPCI in patients with STEMI. We strongly recommend that heliports are located close to the treating facility. Transfer from landing site to hospital by ground ambulance seems unfeasible in time critical patients.

Conflict of interest None declared.

Funding None declared.

\section{COMPREHENSIVE EFFICACY AND INTEGRATED SAFETY RESULTS FROM THE LATE-PHASE CLINICAL PROGRAM OF THE SUFENTANIL SUBLINGUAL TABLET 30 MCG}

${ }^{1} \mathrm{R}$ Leto*, ${ }^{2} \mathrm{~J}$ Miner, ${ }^{3} \mathrm{Z}$ Rafique, ${ }^{4} \mathrm{M}$ Tyler. ' University Hospitals of Leuven, Leuven, Belgium; ${ }^{2}$ Hennepin County Medical Centre; ${ }^{3}$ Baylor College of Medicine; ${ }^{4}$ Macksville Hospital, New South Wales, Austrailia

\subsection{6/bmjopen-2017-EMSabstracts.40}

Aim The sufentanil sublingual tablet system (Zalviso) is a noninvasive, patient-controlled analgesia product recently approved in Europe for treatment of acute post-operative pain. A second sufentanil product, a $30 \mathrm{mcg}$ tablet (SST $30 \mathrm{mcg}$ ) dispensed sublingually by a healthcare professional, recently completed Phase 3 development for treatment of pain in settings such as the emergency department and EMS. The primary objective of this presentation will be to review key efficacy and safety data from all four late-phase trials.

Methods The late-phase program consisted of two randomised, placebo-controlled studies and two, open-label studies, including one of patients presenting to the emergency department with trauma or injury pain. In all studies there was a minimum re-dosing interval of $60 \mathrm{~min}$ and efficacy was assessed using an 11-pt (0-10) NRS. The primary variable was the summed pain intensity difference to baseline (SPID) and safety was evaluated via adverse events (AEs), vital signs and concomitant medications.

Results A total of 479 patients were enrolled; 363 received treatment with SST $30 \mathrm{mcg}$. In each of the placebo-controlled studies, SST $30 \mathrm{mcg}$ was superior to placebo for the primary efficacy endpoint of SPID12 ( $p=0.005$ and $p<0.001$, respectively) while the open-label studies demonstrated clinically significant reductions in pain intensity as early as $15 \mathrm{~min}$ after dosing. The most commonly reported AEs across all studies were nausea (29\%), headache $(8 \%)$ and vomiting (6\%).

Conclusion The sufentanil sublingual tablet $30 \mathrm{mcg}$, while still under FDA review, has shown potential benefit as a non-invasive analgesic modality in medically supervised settings requiring short-term treatment of acute moderate-to-severe pain.

Conflict of interest Drs. J. Miner and Z. Rafique were principal investigators in the phase 3 clinical trial. Dr. R. Leto and M. Tyler have served as consultants to AcelRx Pharmaceuticals Funding Development of the Sufentanil Sublingual Tablet 30 mcg is funded in part by the Clinical and Rehabilitative Medicine Research Program (CRMRP) of the U.S. Army Medical Research and Materiel Command (USAMRMC) under contract No. W81XWH-15-C-0046. The CRMRP was established in 2008 to foster research and technology advances for regeneration, restoration, and rehabilitation of traumatic injuries.

\section{NEW METHODS FOR DIAGNOSIS OF THORACIC TRAUMA IN PREHOSPITAL CARE}

${ }^{1,2,3}$ S Candefjord ${ }^{*},{ }^{1,2,3} \mathrm{R}$ Buendia, ${ }^{4,5} \mathrm{EC}$ Caragounis, ${ }^{6,7} \mathrm{NP}$ Oveland, ${ }^{1,2,3} \mathrm{BA}$ Sjöqvist, ${ }^{8} \mathrm{M}$ Oropeza-Moe, ${ }^{2,9} \mathrm{M}$ Elam, ${ }^{1,2} \mathrm{M}$ Persson, ${ }^{1,2} \mathrm{~A}$ Fhager. ${ }^{1}$ Department of Signals and Systems, Chalmers University of Technology, Gothenburg, Sweden; ${ }^{2}$ MedTech West, Sahlgrenska University Hospital, Gothenburg, Sweden; ${ }^{3}$ SAFER Vehicle and Traffic Safety Centre at Chalmers, Gothenburg, Sweden; ${ }^{4}$ Trauma Unit, Department of Surgery, Sahlgrenska University Hospital, Gothenburg, Sweden; ${ }^{5}$ Department of Surgery, Institute of Clinical Sciences, Sahlgrenska Academy, University of Gothenburg, Gothenburg, Sweden; ${ }^{6}$ Department of Health Studies, Network for Medical Sciences, University of Stavanger, Stavanger, Norway; ${ }^{7}$ Department of Anesthesiology and Intensive Care, Stavanger University Hospital, Norway; ${ }^{8}$ Department of Production Animal Clinical Sciences, Faculty of Vetbio, Norwegian University of Life Sciences, Sandnes, Norway; ${ }^{9}$ Clinical Neurophysiology, Sahlgrenska University Hospital, Gothenburg, Sweden

\subsection{6/bmjopen-2017-EMSabstracts.41}

Aim Trauma to the thorax is common and can be life-threatening. Prehospital diagnosis of thoracic injuries is challenging. Ultrasound is a promising technology; however, its accuracy is operator dependent. Methods not requiring operator image interpretation would be beneficial. The aim of this study is to evaluate electrical bioimpedance (EBI) and microwave technology (MWT). Both technologies are non-operator dependent, non-invasive, harmless, cost efficient, rapid and portable.

Methods Two complementary lines of research are pursued. A clinical study aiming to differentiate EBI measurements of thoracic trauma patients $(n=20)$ and healthy controls $(n=20)$, using diagnostic mathematical algorithms, has been completed. Clinical trials are complemented by experiments on realistic porcine models of pneumothorax (PTX) and hemothorax (HTX). ${ }^{1-2}$ These experiments enable analysis of EBI and MWT with well-defined injuries. A pilot study on two pigs with unilateral PTX from small $(50 \mathrm{~mL})$ to large $(2000 \mathrm{~mL})$ sizes, and large HTX, was performed. Diagnostic performance is evaluated using cross-validation to derive the area under the ROC curve (AUC), and confusion matrices.

Results The clinical study achieved AUC $=0.87$. The pilot porcine study showed that EBI parameters evolved as expected with increasing PTX/HTX; EBI theory predicts presence of air should increase resistivity and fluid decrease it. The MWT classification accuracy for predicting size of PTX was $100 \%$ and 98\% for each pig, respectively.

Conclusion EBI and MW are promising technologies for prehospital diagnosis of thoracic injuries.

\section{REFERENCES}

1. Buendia, et al. Electrical bioimpedance for diagnosing thoracic injuries: Test on a porcine pneumothorax and hemothorax model. (2016) Short Communications From AAAM's 60th Annual Scientific Conference, Traffic Injury Prevention, 17: supp 1, 175-218.

2. Oveland, et al.: A wearable microwave detector for diagnosing thoracic injuriestest on a porcine pneumothorax model. Scandinavian Journal of Trauma, Resuscitation and Emergency Medicine 2015;23(Suppl 2):A20.

Conflict of interest None declared.

Funding The authors acknowledge funding received from the Swedish research centre MedTech West and Folksam insurance company. 